\title{
Article \\ Optimal Power Allocation with Sectored Cells for Sum-Throughput Maximization in Wireless-Powered Communication Networks Based on Hybrid SDMA/NOMA
}

\author{
Juhyun Maeng (D), Mwamba Kasongo Dahouda (D) and Inwhee Joe *(D) \\ Department of Computer Science, Hanyang University, Seoul 04763, Korea; jhmaeng@hanyang.ac.kr (J.M.); \\ dahouda37@hanyang.ac.kr (M.K.D.) \\ * Correspondence: iwjoe@hanyang.ac.kr
}

\section{check for} updates

Citation: Maeng, J.; Dahouda, M.K.; Joe, I. Optimal Power Allocation with Sectored Cells for Sum-Throughput Maximization in Wireless-Powered Communication Networks Based on Hybrid SDMA/NOMA. Electronics 2022, 11, 844. https://doi.org/ 10.3390 /electronics 11060844

Academic Editors: Hirokazu Kobayashi, Pingyi Fan and Andrea Randazzo

Received: 3 January 2022

Accepted: 6 March 2022

Published: 8 March 2022

Publisher's Note: MDPI stays neutral with regard to jurisdictional claims in published maps and institutional affiliations.

Copyright: (c) 2022 by the authors. Licensee MDPI, Basel, Switzerland. This article is an open access article distributed under the terms and conditions of the Creative Commons Attribution (CC BY) license (https:/ / creativecommons.org/licenses/by/ $4.0 /)$.

\begin{abstract}
Wireless-powered communication networks (WPCNs) consist of wireless devices (WDs) that transmit information to the hybrid access point (HAP). In this situation, there is interference among WDs that is considered to be noise and causes information loss because of adjacent signals. Moreover, power is limited and can be lost if transmission distance is long. This paper studies sum-throughput maximization with sectored cells for WPCN. We designed a downlink (DL) energy beamforming by sector based on the hybrid space division multiple access (SDMA) and nonorthogonal multiple access (NOMA) approach to maximize the sum throughput. First, a cell is divided into several sectors, and signals from each sector are transmitted to each antenna of the HAP, so that the signals are not adjacent. Further, the HAP decodes the overlapping information of each sector. Next, power allocation is optimized by sector. To optimize power allocation, a constrained optimization problem is formulated and then converted into a nonconstraint optimization problem using the interior penalty method. The optimal solution derives the maximal value to the problem. Power for each sector is optimally allocated according to this optimal solution. Under this consideration, sum-throughput maximization is performed by optimally allocating DL energy beamforming by sector. We analyzed sum throughput and fairness, and then compared them according to the number of sectors. Performance results show that the proposed optimal power allocation by sector using hybrid SDMA/NOMA outperforms the existing equal power allocation by sector in terms of the sum throughput while fairness is also maintained. Moreover, the performance difference between the hybrid approach and SDMA, which optimally allocates power by sector, was about 1.4 times that of sum throughput on average, and the hybrid approach was dominant. There was also no difference in fairness performance.
\end{abstract}

Keywords: WPCN; sector; power; optimization; hybrid SDMA/NOMA; beamforming

\section{Introduction}

Traditional cellular base stations radiate power in all directions because they have no information about where the mobile device is located; however, a multiantenna technique improves spectral efficiency, range, and bandwidth for mobile wireless devices. Global mobile interoperability for microwave access and long-term evolution networks is expected to use spatial division multiple access (SDMA) technologies, which are extensively used in private wireless broadband systems. In this system, a base station's radiation pattern may be changed to maximize both transmission and reception for each user device by utilizing smart antenna technology to track the spatial position of mobile devices. Moreover, the base station can efficiently direct a beam or a spot of radio frequency (RF) power to or from each user by quickly changing the phase of signals from multiple antennas. Unlike multipleinput multiple-output (MIMO), just one antenna at the client device is required, possibly lowering customer premise equipment (CPE) costs. The device's lifetime is restricted in traditional wireless sensor networks (WSNs) since it is powered by a fixed-energy 
battery [1]. In order to extend the network lifetime, energy recovery or battery replacement is periodically required. However, in WSNs, this is difficult [2]. Wireless sensing devices are the core components of Internet of Things (IoT) systems, which have been quickly deployed in both consumer and industrial applications to provide a variety of sensing and control services in recent years. The majority of those sensing devices, on the other hand, are battery-powered, necessitating significant financial and human resources to replace or charge the batteries on a regular basis in order to keep them properly functioning. To address this problem, an increasing number of IoT devices are now fuelled by harvesting energy sources that are often unreliable and difficult to govern. Emerging radio-frequencybased energy harvesting technologies, on the other hand, offer significant advantages in terms of being wireless and controllable in order to provide a reliable source of energy. Because IoT systems rely on radio-frequency-based energy harvesting technologies, the WPCN overcomes such obstacles, and the optimization outcomes are more significant in real-time applications such as IoT systems. Recently, wireless power transfer (WPT) has emerged as a promising technology that can extend a device's lifetime. Wireless powered communication networks (WPCNs) can be built using this WPT. WPCNs consist of the hybrid access point (HAP) and wireless devices (WDs) where the WDs recharge power from HAP [3], and the HAP continuously transmits power to WDs. Through the harvested energy, WDs transmit information to the HAP [4]. If the power of the device is continuously recharged through this WPCN, the lifetime can be expanded. However, a device outside the transmission range may not be able to stably power recharge. Power recharging WD is important because wireless information transfer (WIT) cannot be performed if the power of WD is insufficient. Therefore, beamforming may be used to increase the transmission range [5]. Beamforming or spatial filtering is a signal processing technique used in sensor arrays for directional signal transmission or reception. This is achieved by combining elements in an antenna array in such a way that signals at particular angles experience constructive interference, while others experience destructive interference. Beamforming can be used at both the transmitting and receiving ends in order to achieve spatial selectivity (https:/ / en.wikipedia.org/wiki/Beamforming, accessed on 18 January 2021). In wireless communication, beamforming can be divided into three categories: analog, digital, and hybrid beamforming.

\subsection{Analog Beamforming}

In analog beamforming (ABF), the traditional way to form beams is to use attenuators and phase shifters as part of the analog RF circuitry where a single data stream is divided into separate paths. Only one beam is created for the entire frequency band, which is sufficient for line-of-sight (LoS) beamforming [6]. The advantage of this method is that only one RF chain is required. The disadvantage is the loss from the cascaded phase shifters at high power.

\subsection{Digital Beamforming}

Digital beamforming (DBF) assumes that there is a separate RF chain for each antenna element. The beam is formed by matrix-based operations in the baseband where digitalamplitude and phase weighting is performed. In digital beamforming, amplitude/phase variation is applied to the digital signal before the digital-to-analog converter (DAC) at the transmitting end. The reverse process is conducted after analog-to-digital converter (ADC) and digital down converter (DDC) operations are performed. DBF designs different signals for each antenna in the digital baseband; in other words, different signals are designed for each antenna in the digital domain. This allows for greater flexibility since one can assign different powers and phases to different antennas and different parts of the frequency bands. This means that digital beamforming is particularly desirable for spatial multiplexing where we want to transmit a superposition of signals, each with separate directivity. 


\section{Digital Beamforming Challenges}

The design of broadband microwave-phased array digital beamformers for SKAPhase 1 presents several technological problems [7] such as bandwidth, radio-frequency interference (RFI), cooling, coherency, and complexity, in addition to financial and timing constraints. (1) Amount of generated data: the data rate out of the ADC affects the digital interface and processing power requirements, and the question becomes how to handle the data when electronic systems need increased resolution and a higher sampling rate for increased bandwidth. (2) Power consumption: Processors require much power. Because of the limitation in data bandwidth, there is a practical limit on the number of elements in the array which requires waveform generators at each element. (3) Loss: losses that high-frequency mmWave transmission incurs include high free space path loss, absorption from atmospheric gases and rainfall, and non-line-of-sight propagation. (4) Expense: the overall expense of implementing digital beamforming systems includes the physical size of the electronics and the high cost of a large number of ADCs operating at high sampling frequencies.

\subsection{Hybrid Beamforming}

Hybrid beamforming (HBF): HBF combines DBF with ABF in order to allow for the flexibility of MIMO plus beamforming while reducing the cost and losses of the beamforming unit (BFU). Each data stream has its own analog BFU with a set of $\mathrm{M}$ antennas. If there are $\mathrm{N}$ data streams, then there are $N \times M$ antennas. The analog BFU loss due to phase shifters can be mitigated by replacing the adaptive phase shifters with a selective beamformer such as a butler matrix.

\subsection{Difference between Analog and Digital Beamforming}

Due to substantial channel losses and high energy consumption by ADC, which increases with bandwidth [8], energy is a precious resource in mobile millimeter wave (mmWave) systems. In the ABF type, amplitude/phase variation is applied to an analog signal at the transmitter end. At the receiver end, signals from different antennas are summed up before ADC conversion. ABF uses phase shifters to send the same signal from multiple antennas but different phases; in other words, the same signal is fed to each antenna, and analog phase shifters are then used to steer the signal emitted by the array.

The module configuration of digital/hybrid beamforming is complicated, and power consumption increases due to complex processing. Power management is important because WPCN is a resource constraint. Therefore, we used analog beamforming, which consumes less power because of its relatively simple module configuration and simple processing. In addition, the power transmission range is expanded by beamforming to ensure the power recharge of the device far from the HAP. Under these considerations, DL energy beamforming by sector is optimized for sum-throughput maximization. In addition, hybrid SDMA and NOMA approaches are proposed to reduce interference between multiple WDs that reduce sum throughput. Our contributions can be summarized as follows: (1) we propose optimal power allocation by sector using analog beamforming based on the hybrid SDMA and NOMA approach that mixes SDMA and NOMA for sumthroughput maximization; (2) we transmitted more power to sectors containing many WDs with a short distance to HAP in order to optimize power allocation by sector. This is because minimizing power loss due to long distances can increase the total amount of power transmitted with a sectored cell and improve sum throughput; (3) we perform information reception by sector from each antenna of the HAP to reduce interference between multiple WDs, and we use NOMA to superimpose signals from multiple WDs. This is because sum throughput can be improved by dividing cells and by separating overlapping signals according to signal strength differences to reduce information loss due to interference between multiple WDs; (4) in performance evaluation, we demonstrated the effectiveness of optimal power allocation, and the hybrid SDMA and NOMA approach. This shows that, in terms of the sum throughput, the proposed optimal power allocation 
by sector based on the hybrid SDMA and NOMA approach outperforms existing equal power allocation by sector while fairness is maintained.

The rest of this paper is organized as follows: Section 2 presents the related work. Section 3 introduces the hybrid SDMA and NOMA approach and the optimal power allocation. Section 4 analyzes various cases of the hybrid SDMA and NOMA approach, and the optimal power allocation in terms of sum throughput and fairness. Lastly, Section 5 concludes the paper.

\section{Related Work}

Recently, interest in WPCNs driven by energy obtained by RF signals has increased [9]. For instance, in [10] the authors integrated wireless power transfer and wireless information transfer in a WPCN in which an unmanned aerial vehicle (UAV) with a continuous power supply coordinated the WPT in the downlink and WIT in the uplink. In addition, the use of UAV as an energy transmitter and data collector in WSNs was studied in [11]. Energy harvesting (EH) to obtain energy from RF signals was proposed as a technology capable of expanding the battery lifetime of devices [12]. The feasibility of the optimization issue and the presence of a simple solution were examined in [13], two-user multiple-input single-output (MISO) for wireless powered communication networks where user devices are equipped with nonlinear EH circuits. As a result, the problem was double and had a nontrivial solution, and they demonstrated that three beamforming vectors were enough for optimal downlink transmission. Therefore, in WPCN, the device could improve battery constraints while continuously supplementing energy through EH. Regarding improving the battery lifetime of the device, various studies were conducted for sum-throughput maximization in WPCN. In [14], the author proposed a mesh-topology-based wireless-powered communication network (MT-WPCN) for sum-throughput maximization. In the MT-WPCN, each user controls the transmission power and schedules energy for receiving information. In addition, the author studied half harvest-then-transceive and full harvest-then-transceive. NOMA is one of the most promising radio access techniques in next-generation wireless communications (https://www.springeropen.com/collections/noma, accessed on 14 October 2021). When compared to the current de facto standard orthogonal multiple access (OMA) technology, orthogonal frequency division multiple access (OFDMA), NOMA has a number of appealing potential advantages, including increased spectral efficiency, low latency with high dependability, and enormous connection. NOMA's fundamental concept is to service numerous consumers at the same time, frequency, and location. Recent studies [15-21] demonstrated that NOMA could be applied in various fifth-generation (5G) communication scenarios, including machine-to-machine (M2M) communications and the Internet of Things (IoT). The performance of two-tier NOMA-based wireless powered communication networks was investigated [22], and the distant user suffered from the doubly near-far effect. To address this problem, an adaptive energy-transfer time method was devised by optimizing the far user's data rate and determining the best solution. NOMAbased WPCN using cluster-specific beamforming was considered in [23]. NOMA-based WPCN groups users into multiple clusters according to cluster-specific beamforming. Time and energy resources were optimized for beamforming determined by signal alignment. The NOMA decoding technique was studied in [24], and for sum-throughput maximization, two decoding techniques were proposed depending on whether the receiver side had successive interference cancellation (SIC). In addition, time and power allocation were optimized. Optimal resource allocation in a WPCN combining time reverse (TR) was studied in [25], and sum throughput was improved by optimizing the time allocation of HAP for DL WET, DL TR, and UL WIT in TR-WPCN. When compared to time division multiple access (TDMA), SDMA for multiantenna broadcast channels offers much potential in terms of increasing system capacity. In [26], the authors developed a novel codebook-based SDMA system with a configurable number of active beams, and they proved that, in sparse networks, the suggested SDMA scheme outperforms several existing SDMA schemes in terms of sum throughput while having comparable feedback cost. SDMA techniques were 
studied in [27-32], and SDMA increases the available spectral efficiency, range, and bandwidth to moving wireless devices. In [33], the author proposed a hybrid approach based on multiuser multi-input single-output (MU-MISO) to improve the existing SDMA. As the interference between multiple WDs is reduced through spatial multiplexing and NOMA, sum throughput is improved. However, WPCN is suitable for low-power applications such as WSN, constrained resource management is very important. Therefore, optimal resource allocation from HAP for sum-throughput maximization is required.

This paper proposes optimal power allocation combining hybrid SDMA and NOMA approach by sector using model-based analog beamforming. We initially investigate minimizing interference between multiple WDs in order to maximize the sum throughput of information received in the WPCN. As a result, a cell is divided into many sectors, and the HAP obtain superposed information from several WDs in each sector for each antenna using the hybrid SDMA and NOMA approach.

\section{System Model}

\subsection{Proposed System Model}

In this paper, we assumed that, as shown in Figure 1, the system model was composed of one HAP at the center and many WDs within a cell, where $W D_{i}, \forall i \in 1,2, \ldots, W$ represents the wireless device. The cell was also divided into multiple sectors of the same size. The HAP was equipped with multiple antennas for allocating power per sector by DL energy beamforming and for receiving overlapping information from each sector. All WDs were equipped with one antenna for harvesting the energy transmitted by the HAP and for transferring information wirelessly to the HAP. In this case, the HAP and all WDs used a directional antenna. The HAP and all WDs use the same frequency band. As shown in Figure 1, the wireless energy transfer (WET) between HAP and all WDs and the channel gain for WIT was the same and is expressed as $H_{i}, \forall i \in 1,2, \ldots, W$. The channel used a quasistatic flat-fading model and is constant for each block transmission time indicated by T. HAP was fully aware of channel state information (CSI). The power transmitted from the HAP to each sector by DL energy beamforming is represented by $H_{p}$. In addition, noise affects the transmitted power and is represented by $N_{i}, \forall i \in 1,2, \ldots, W$. The distance between HAP and $W D_{i}$ in each sector is expressed as $D_{i}, \forall i \in 1,2, \ldots, W$. Path loss only takes into account the loss caused by the distance between the two antennas on the LoS. The sum of the energy harvested by $W D_{i}, \forall i \in 1,2, \ldots, W$ in each sector is as follows:

$$
S_{p}=\sum_{i=1}^{n} \frac{H_{p} * H_{i}}{N_{i} * D_{i}},
$$

where, $S_{p}$ represents the sum of SINR for one sector, and $n$ is the total number of WDs. In one cell, the sum of $S_{p}, \forall p \in 1,2, \ldots, S$, is as follows:

$$
C_{p}=\sum_{i=1}^{n} S_{p_{i}},
$$

where $C_{p}$ represents the sum of SINRs in one cell. 


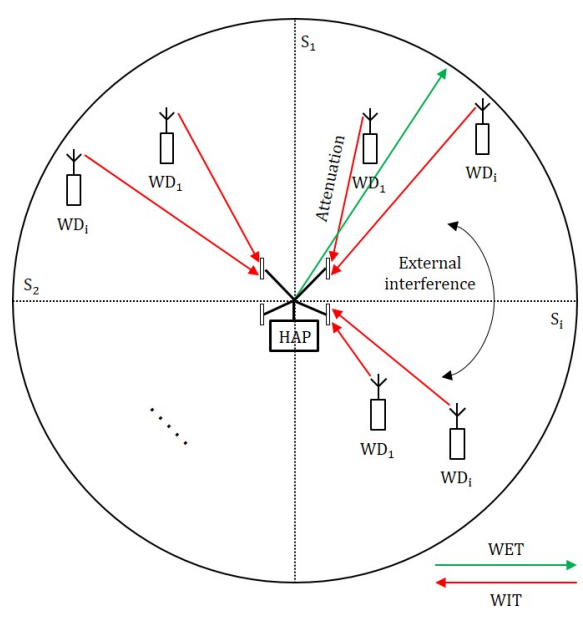

Figure 1. System model: hybrid SDMA and NOMA approach and optimal power allocation.

As shown in Figure 1, one cell was divided into sectors with same size $S_{i}$, $\forall i \in 1,2, \ldots, S$, where $S_{i}$ represents each sector. Moreover, $S_{\theta i}$ represents an angle of each sector as described in Figure 2a. As shown in Figure $2 b, W D_{i}, \forall i \in 1,2, \ldots, W$ was distributed in the cell according to $W_{\theta i}$ and $D_{i}, \forall i \in 1,2, \ldots, W$, where $W_{\theta i}$ represents an angle of $W D_{i}$.

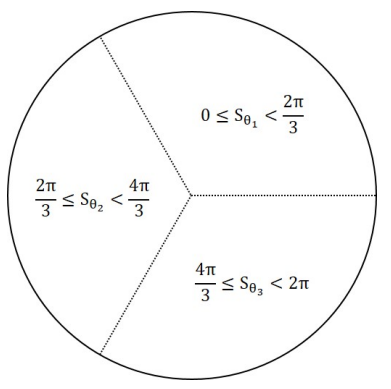

(a)

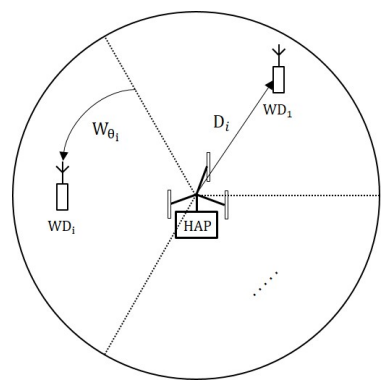

(b)

Figure 2. (a) Sector division. (b) Distance and angle of WD from HAP.

As shown in Figure 3, the network applies harvest-then-transmit protocol [4] and half-duplex. During each block transmission time, the HAP sequentially transmits power to each sector by analog beamforming at the same strength. HAP is stably supplied with energy, and all WDs harvest energy in each sector. Then, all WDs in the cell simultaneously transfer information to the HAP while consuming all of the harvested energy. The HAP receives information of each sector with each antenna. The TDMA structure is used for HAP to sequentially perform WET with each sector by analog beamforming. In each time slot, all WDs for each sector perform energy harvesting. Here, $\tau_{i}, \forall i \in 1,2, \ldots, S$ represents the time of DL energy beamforming allocated to each sector, and the sum of time for one cell is expressed as follows:

$$
T=\sum_{i=1}^{S} \tau_{i}, \text { with } T<=1,
$$

where, $S$ represents the total number of sectors in which one cell is divided. According to optimization, $\tau_{i}$ for each sector is allocated differently, and optimization occurs periodically. After that, during the allocated time, all WDs in each sector transfer information, and the HAP receives information in the maximal time, which is $\max \left(\tau_{i}\right), \forall i \in 1,2, \ldots, S$. Since HAP receives information for each sector at the same time; therefore, it receives information during $\max \left(\tau_{i}\right)$ allocation to the sector that wirelessly transfers information for the longest time. 


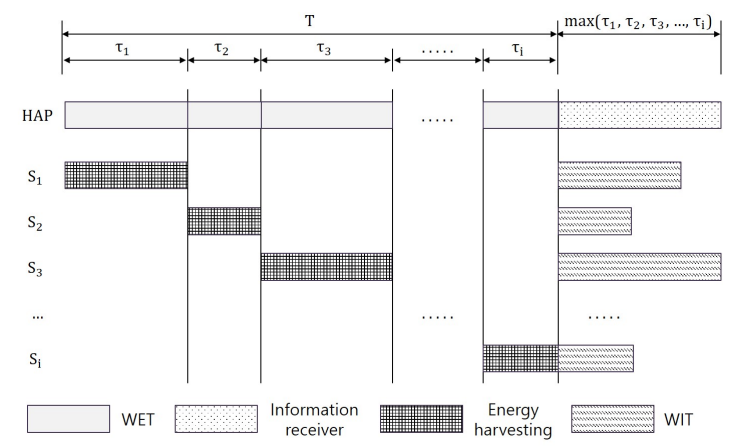

Figure 3. Harvest-then-transceive protocol in hybrid SDMA and NOMA approach and optimal power allocation.

\subsection{Hybrid SDMA/NOMA Approach}

In this section, we refer to the proposed hybrid approach that combines SDMA and NOMA. As shown in Figure 4, the overlapping information of each sector $S_{i}, \forall i \in 1,2, \ldots, S$ is transmitted to each antenna $A_{i}, \forall i \in 1,2, \ldots, A$, of the HAP. SDMA is used to receive information for each sector from each antenna of the HAP while NOMA is applied for multiple WDs to simultaneously transmit information to HAP in the same spectrum [34]. During $\max \left(\tau_{i}\right), \forall i \in 1,2, \ldots, S$, taking into account the attenuation effect over distance, WD close to HAP wirelessly transfers information with weak signal strength while the WD far from HAP transfers information wirelessly with strong signal strength. Figure 5 shows how the HAP decodes the overlapping information $Y_{i}, \forall i \in 1,2, \ldots, W$ received by the HAP from each sector, and the overlapping information is expressed as follows:

$$
Y_{i}=h_{i} x_{i}+N_{i}, \forall i \in 1,2, \ldots, W,
$$

where, $h_{i}, \forall i \in 1,2, \ldots, W$ represents the channel gain, and $x_{i}, \forall i \in 1,2, \ldots, W$ is the information transmitted by each WD. $N_{i}, \forall i \in 1,2, \ldots, W$ is the additive white Gaussian noise (AWGN).
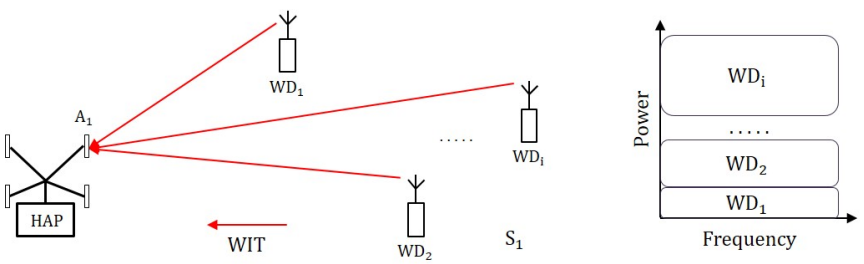

Figure 4. System model: NOMA.

SIC is applied to separate the overlapping information of each sector received by the HAP according to the difference in signal strength. As shown in Figure 5, after decoding the strong signal of $Y_{1}$, weak signal $x_{1}$ is first extracted and removed; after that, the weak signal from $x_{2}$ to $x_{i}$ is sequentially extracted and removed by the same procedure.

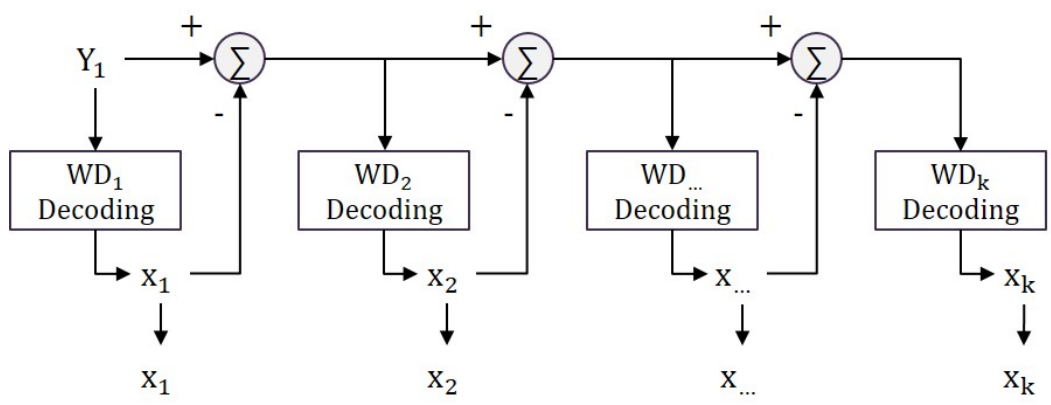

Figure 5. System model: SIC. 
The hybrid SDMA and NOMA approaches reduce interference between each signal transmitted to the HAP, which means all WDs simultaneously wirelessly transfer information to the HAP. As shown in Algorithm 1, we divided one cell into several sectors, placed multiple WDs in each sector, and expressed the procedure for setting the initial SINR for each WD.

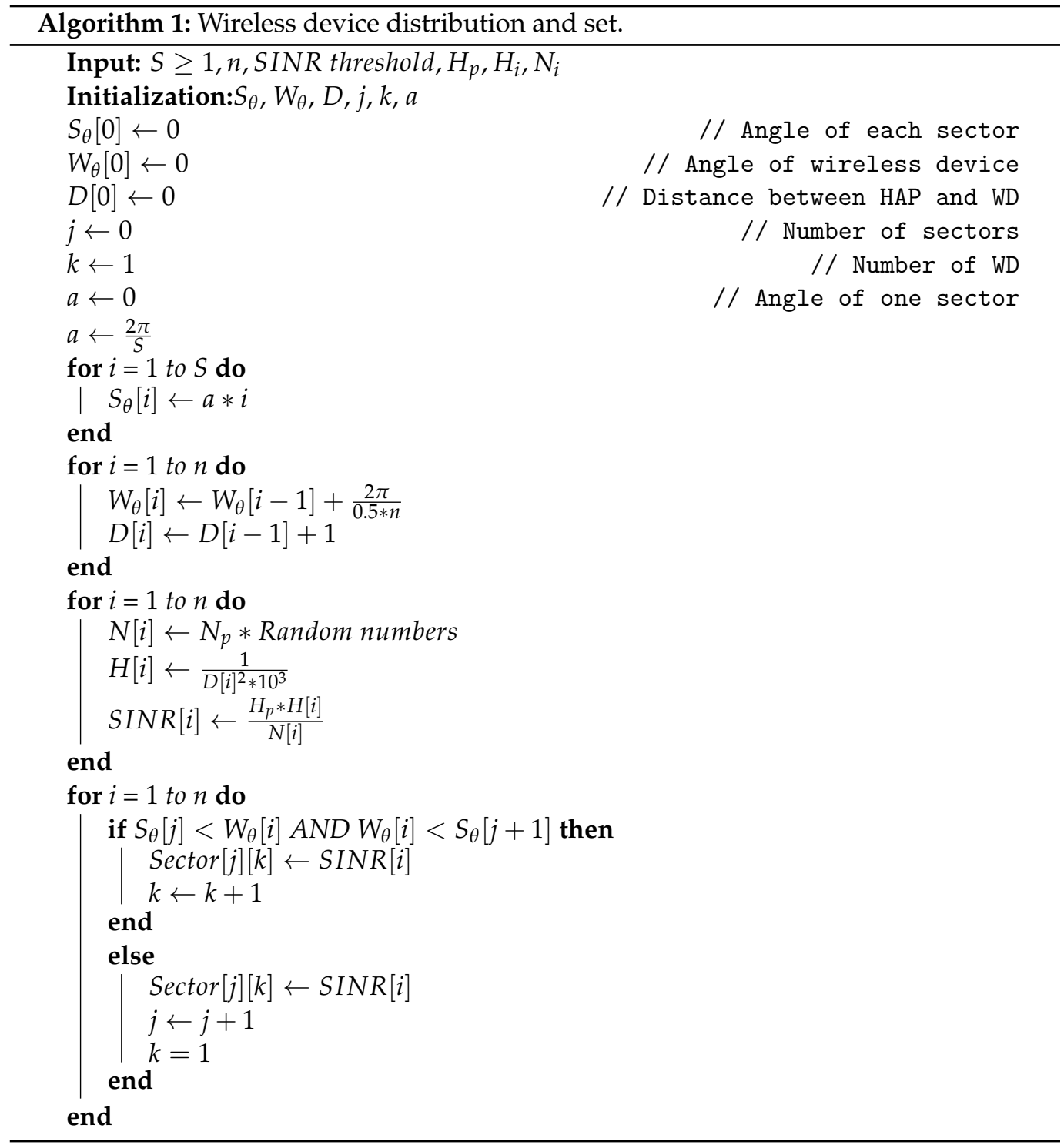

As shown in Algorithm 2, the procedure for optimizing power allocation by sector, and calculating sum throughput and fairness is expressed. 


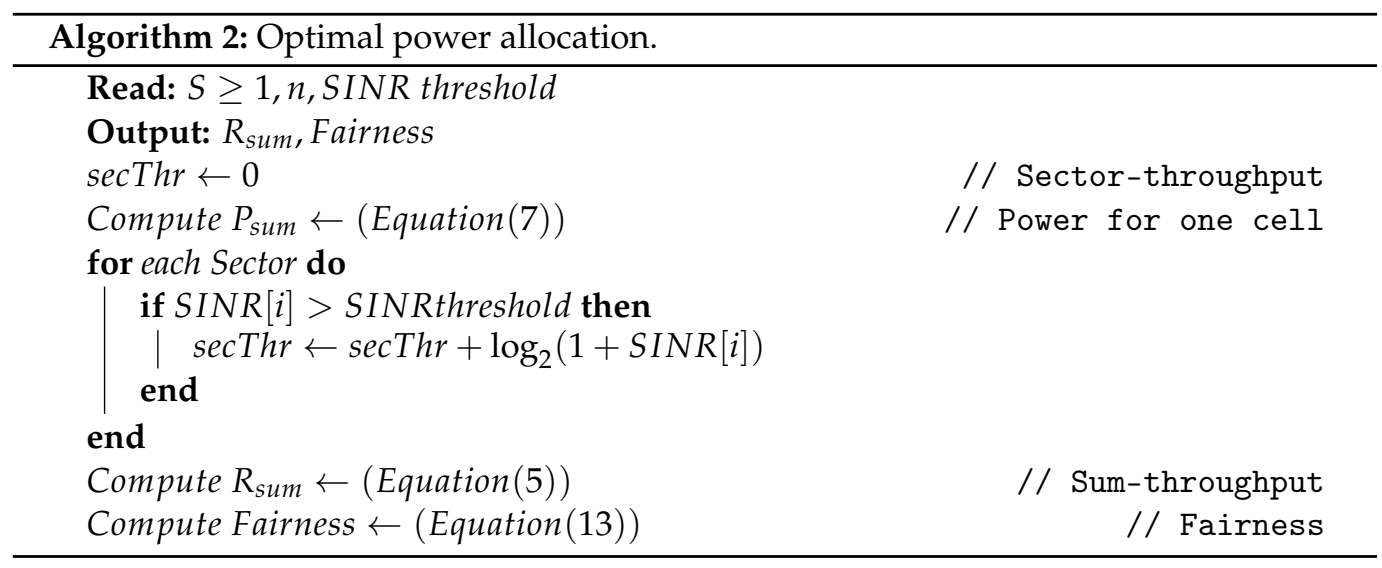

\subsection{Optimal Power Allocation}

We study sum-throughput maximization in the hybrid SDMA and NOMA approach. Sum throughput measured by HAP is expressed as follows:

$$
\begin{gathered}
R_{\text {sum }}=\sum_{i=1}^{S} S_{i}, \\
S_{\text {sum }}=\sum_{i=1}^{n} \log _{2}\left(1+\operatorname{SINR} R_{i}\right)
\end{gathered}
$$

where $R_{\text {sum }}$ is the sum throughput, $S$ is the total number of sectors, and $S_{\text {sum }}$ is the sector throughput, and $n$ is the total number of WDs. Here, the channel capacity is used to calculate the throughput of each WD, and the SINR which is represented by $\operatorname{SINR}_{i}, \forall i \in 1,2, \ldots, W$, for WIT of each WD is affected by WET of HAP. That means if the WET is large then the throughput is increased due to the increased SINR. Therefore, the power allocation for each sector is optimized for sum-throughput maximization, which is expressed as follows:

$$
\begin{gathered}
\max _{\tau} P_{\text {sum }}=\sum_{i=1}^{S} \tau_{i} S_{i}, \\
\max _{\tau} P_{\text {sum }}=\sum_{i=1}^{S} \tau_{i} \sum_{j=1}^{n} \frac{H_{p} * H_{j}}{N_{j} * D_{j}}, \\
\text { s.t. } 0 \leq \tau_{i} \leq 1, \forall i \in 1,2, \ldots, S, \\
\text { s.t. } \sum_{i=1}^{n} \tau_{i} \leq 1,
\end{gathered}
$$

where $P_{\text {sum }}$ represents the sum of power for one cell, and $\tau_{i}, \forall i \in 1,2, \ldots, S$ is the time of DL energy beamforming for each sector. The interior penalty method is used to optimize power allocation by sector [35]; therefore, our constrained optimization problem is first converted into an unconstrained optimization problem, and it is expressed as follows:

$$
\phi\left(X, r_{k}\right)=f(X)+r_{k} \sum_{i=1}^{n} \frac{1}{g_{i}(X)}
$$

where, $f(X)$ represents $P_{\text {sum }}$, which is an objective function, and $r_{k} \sum_{i=1}^{n} \frac{1}{g_{i}(X)}$ represents a penalty term. In order to find optimal solution $(X)$ for deriving maximal value $r_{k} \forall k \in$ $1,2, \ldots, K$ is sequentially reduced. The penalty term prevents the maximal value from crossing the boundaries of constraints, and $g_{i}(X)$ is converted according to constraints in Equation (8) as follows:

$$
g_{i}(X)=x_{1}+x_{2}+\ldots+x_{i}-1 \leq 0,
$$




$$
B(X)=-\frac{1}{g_{i}(X)}=\frac{-1}{x_{1}+x_{2}+\ldots+x_{i}-1},
$$

Optimal solution $X$ satisfies all constraints. Next, the transformed unconstrained optimization problem is expressed as follows:

$$
\begin{aligned}
T\left(X, r_{k}\right)= & {\left[\sum_{i=1}^{S} \tau_{i} \sum_{j=1}^{n} \frac{H_{p} * H_{j}}{N_{j} * D_{j}}\right]+r_{k}\left(\frac{1}{x_{1}}-\ldots+\frac{1}{x_{i}}\right.} \\
& -\frac{1}{x_{1}-1}-\ldots-\frac{1}{x_{i}-1} \\
& \left.-\frac{-1}{x_{1}+x_{2}+\ldots+x_{i}-1}\right) .
\end{aligned}
$$

Lastly, the power for each sector is optimally allocated according to optimal solution $(X)$ that derives the maximal value.

\section{Performance Evaluation}

In this section, we show the results of analyzing the optimal power allocation for each sector on the basis of the proposed hybrid SDMA and NOMA approach. The values used in the experiment are the same as in Table 1 where $S_{i}, W D_{i}, S I N R_{t h}$, and $H_{p}$ are for the whole system, $H_{i}$ and $N_{i}$ are for each sector. Performance evaluation results represent the average value of the results of 100 experiments in a graph.

Table 1. Simulation parameter values.

\begin{tabular}{cc}
\hline Symbol & Value \\
\hline$S_{i}$ & Variable \\
$W D_{i}$ & 100 \\
$S I N R_{t h}$ & Variable \\
$H_{p}$ & $30 \mathrm{dBm}$ \\
$H_{i}$ & $10^{-3} \times D_{i}^{-2}$ \\
$N_{i}$ & $-30 \mathrm{dBm} \times$ Random numbers \\
\hline
\end{tabular}

Sum throughput is calculated according to Equations (5) and (6), described above. This sum throughput represents the sum of sector throughput, and Jain's fairness is used to calculate the fairness [36]. Fairness evaluates how fair throughput per sector is. In other words, the more throughput per sector is equal, the closer the fairness is equal to 1 ; however, the larger is the difference, the closer the fairness is equal to 0 . The fairness is expressed as follows:

$$
\text { Fairness }=\frac{\left(\sum_{i=1}^{S} S_{t h[i]}\right)^{2}}{S\left(\sum_{i=1}^{S} S_{t h[i]}^{2}\right)^{\prime}}
$$

where $S$ represents the total number of sectors in which a cell is divided, and $S_{t h[i]}, \forall i \in$ $1,2, \ldots, S$ is the sum of each WD throughput for each sector. Performance evaluations show that optimal power allocation for each sector based on the sector model and reduction of interference through the hybrid approach can maximize sum throughput while maintaining fairness

\subsection{Performance Comparison}

Figures 6 and 7 are the results of comparing the performance of the hybrid approach and SDMA depending on whether or not the optimization. 


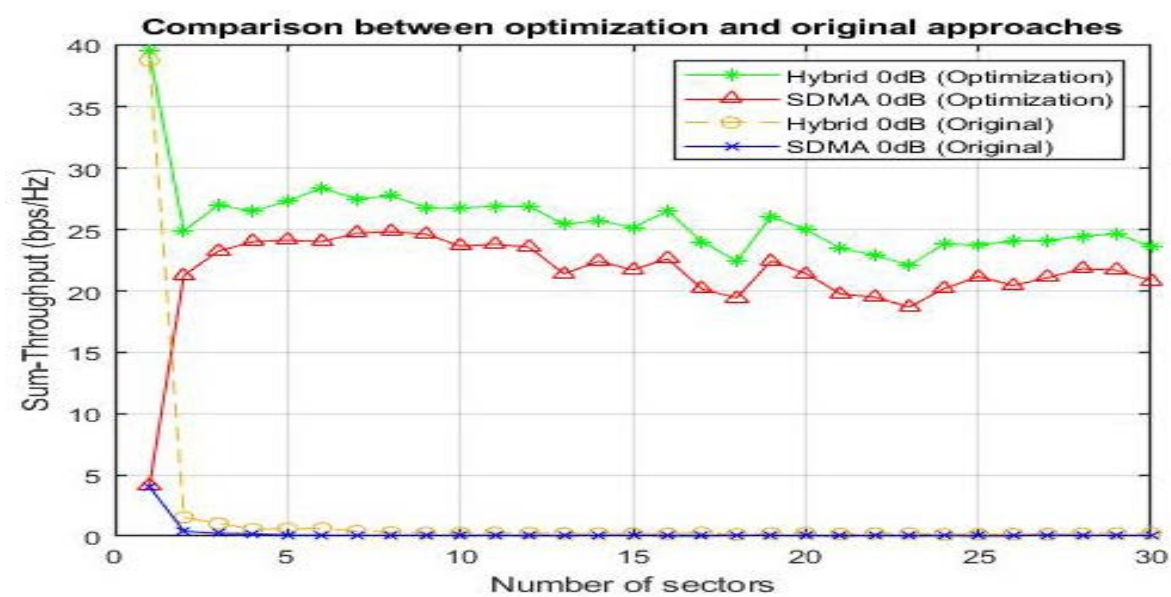

Figure 6. Sum throughput: comparison between optimization and original approaches.

Figure 6 shows the results of sum throughput according to the number of sectors. Optimal power allocation by sector and hybrid approach improve sum throughput. Because equal power allocation by sector increases losses by equally transmitting power to sectors with many WDs with long distances between HAP and WD. In addition, SDMA does not relatively reduce interference between WDs; however, the optimal power allocation for each sector can transmit more power to a sector with many WDs with a short distance between HAP and WD. Since this minimizes the loss of power according to the distance, it increases the total amount of power transmitted to one cell. In addition, the hybrid approach divides the area and simultaneously reduces interference between WDs using NOMA. This reduces information loss due to interference between multiple WDs by performing information reception by sector with each antenna of the HAP and separating the overlapping information according to the difference in signal strength. As a result, sum throughput is improved, and the proposed hybrid approach outperformed the existing SDMA. As shown in Figure 6, the difference of performance between the H hybrid approach and SDMA for optimal power allocation was about 1.4 times on average, and the hybrid approach substantially improved sum throughput compared to SDMA. Since the factor that most influences sum throughput is the distance between HAP and WD, the trend of changes in sum throughput according to the number of sectors may not be constant. The hybrid approach is more energy efficient compared to SDMA. NOMA of the hybrid approach multiplexes the signals of multiple WDs per sector in the power domain. As a result, the interference between signals is reduced and the transmission rate is improved. In addition, the energy consumption of each WD can be reduced because retransmissions are reduced. Figure 7 shows the results of fairness according to the number of sectors. Optimal power allocation by sector and hybrid approach maintain fairness while the performance of the hybrid approach and SDMA is similar. As shown in Figure 7, there was no difference in performance between the hybrid approach and SDMA, which optimally allocated power for each sector.

As the number of sectors increased, performance decreased because, as shown in Equation (13), the total number of sectors was inversely proportional to the size of fairness. 


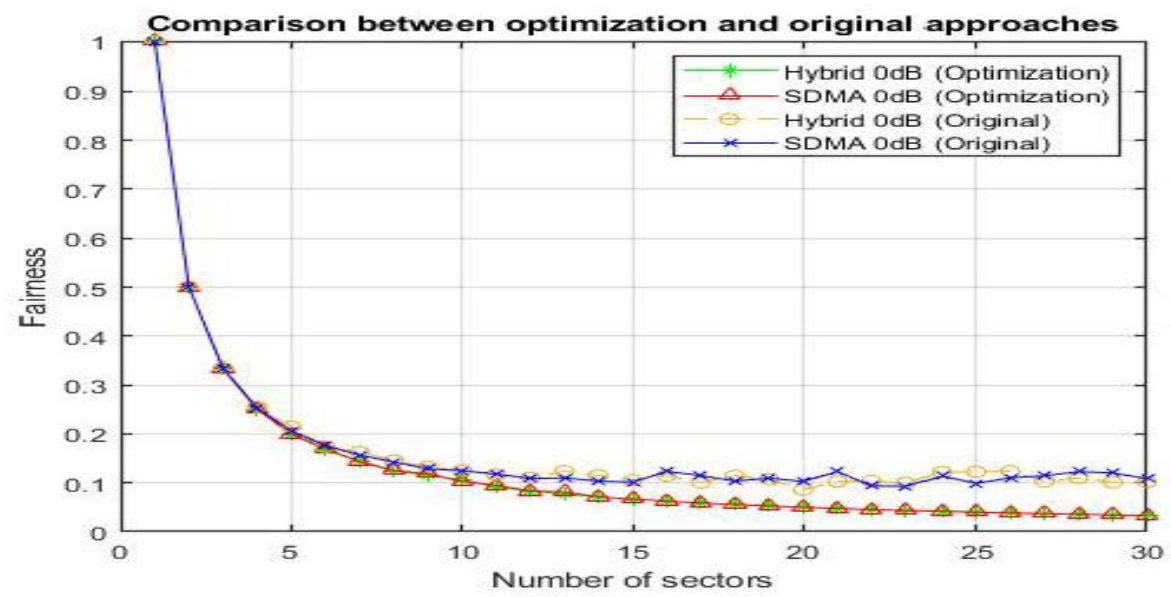

Figure 7. Fairness: comparison between optimization and original approaches.

\subsection{Performance Analysis of Hybrid SDMA/NOMA by SINR Threshold}

Figures 8 and 9 are the results of comparing the performance of the hybrid approach by SINR threshold depending on whether or not the optimization.

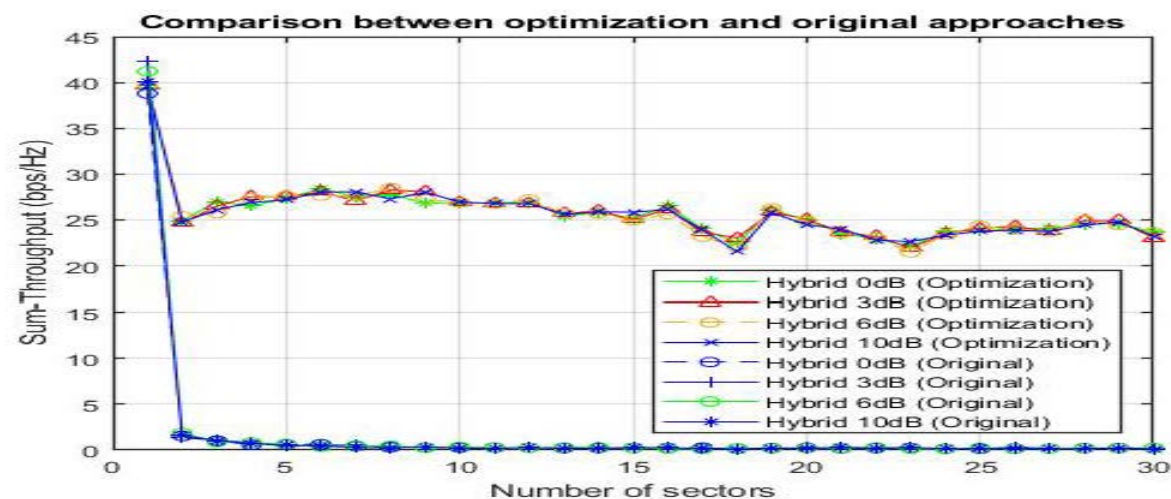

Figure 8. Sum throughput: comparison between optimization and original approaches by SINR threshold.

Figure 8 shows the results of sum throughput according to the number of sectors. The optimal power allocation by sector improves sum throughput and maintains the performance of hybrid approach by SINR threshold. This is because the influence of the SINR threshold decreased, optimally allocating power for each sector according to the distance difference between HAP and WD.

Figure 9 shows the results of fairness according to the number of sectors. Both the optimal power allocation by sector and the hybrid approach by SINR threshold maintained fairness because the influence of the SINR threshold decreased due to the optimal power allocation for each sector. 


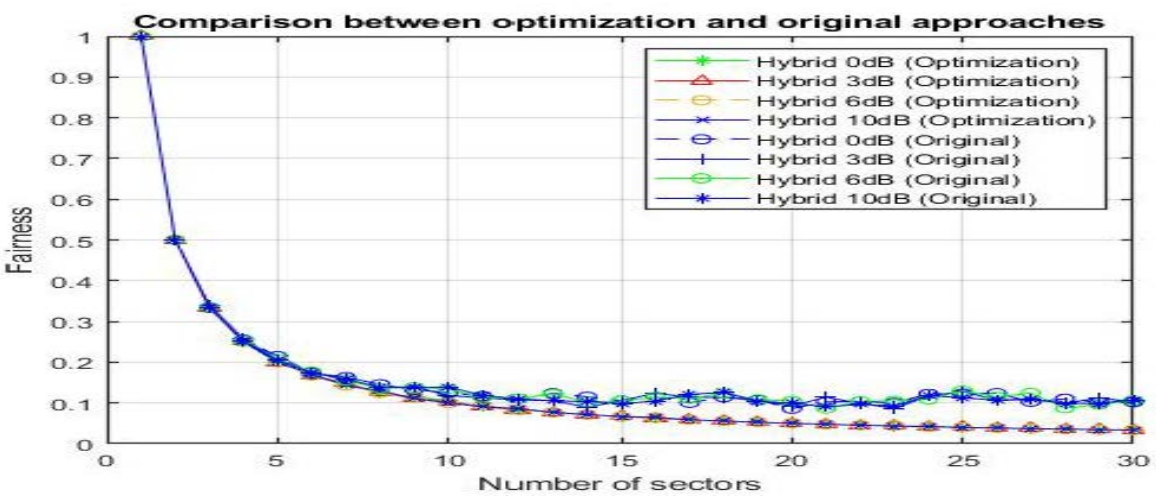

Figure 9. Fairness: comparison between optimization and original approaches by SINR threshold.

\subsection{Tradeoff Analysis of Hybrid SDMA/NOMA}

Figure 10 is the result of analyzing tradeoff that considers sum throughput and fairness for each sector of the hybrid approach depending on whether or not the optimization. Here, only SINR 0dB is considered. The optimal number of sectors of the hybrid approach through optimal power allocation by sector is about 3 .

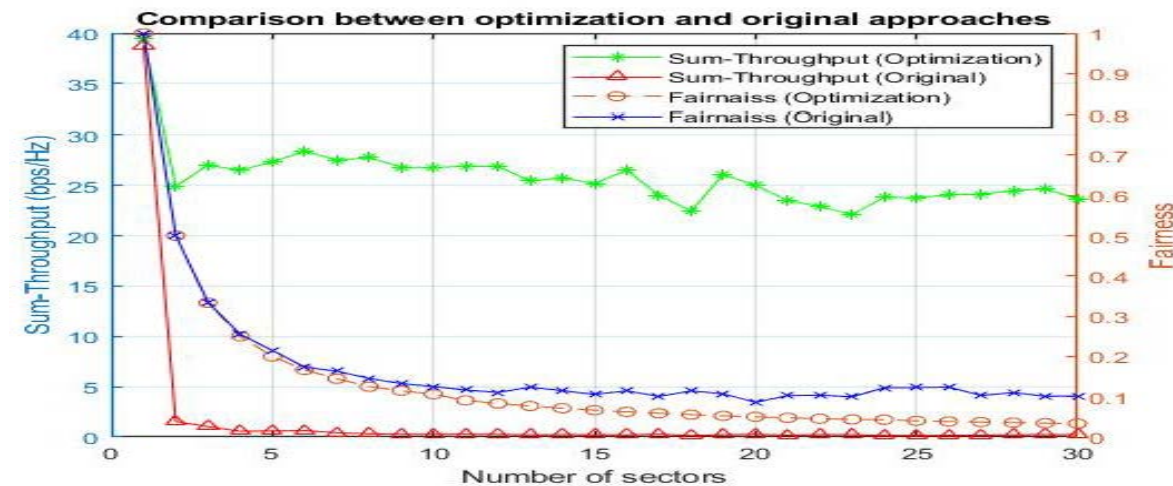

Figure 10. Trade-off: comparison between optimization and original approaches.

\subsection{Computational Cost Analysis}

The computational cost of the proposal for calculating sum throughput can be represented by Big-O notation. Input values that affect the computational cost are the number of WDs (N) and the number of sectors (M). In addition, the optimal power allocation by sector and the transmission rate of each WD are constants. Fairness is a calculated value at maximal sum throughput and is a constant. Sum throughput is calculated by the sum of the transmission rate of each WD and the optimal power allocation by sector affected by the input values $\mathrm{N}$ and $\mathrm{M}$. Since $\mathrm{N}$ is quite large, $\mathrm{M}$ is negligible compared to $\mathrm{N}$. Thus, according to Big-O notation, the computational cost of sum throughput can be approximated to $\mathrm{O}(\mathrm{N})$.

\subsection{Study Limitations}

Several limitations of this study should be mentioned. First, it is necessary to apply the proposed method to the WSN and prove its effectiveness because WPCN is suitable for various low-power applications such as WSNs [3]. In particular, various applications may be implemented by improving the efficiency of interaction between different regions through data collection in WSN. However, various applications may not be implemented as the operation of the WSN becomes unstable due to the battery constraints of the batterydriven device. Therefore, the proposed method proves the improvement of the limitations according to the device's battery constraints, thereby contributing to the improvement of WSN utilization. To this end, the proposed optimal power allocation by sector and hybrid approach in the IEEE 802.15 protocol-based development kit are implemented, and 
performance can be evaluated by randomly deploying the devices in each sector. Because of the optimal power allocation by sector, more power is transmitted to a specific sector, and as the number of sectors increases, fairness between sectors becomes unfair. This is a trade-off that occurs because improving sum throughput is the top priority, but unfairness between sectors needs to be improved. This is because collected data from all devices in the WSN are equally important [37], and the sensing range of each device deployed according to application requirements is different [38-40]. In order to improve fairness between sectors according to the throughput for each sector, joint optimization that maximizes fairness and sum throughput can be implemented.

\section{Conclusions}

This paper proposed optimal power allocation combining hybrid SDMA and NOMA by sector using model-based analog beamforming in WPCN. The HAP performs DL energy beamforming for each sector, and each WD transmits information to the HAP after harvesting energy. We designed optimal power allocation using hybrid SDMA and NOMA. In WPCN, we considered optimal power allocation by sector and interference reduction between multiple WDs that simultaneously transmit information to the HAP. First, sectors were divided in order to reduce interference, and superimposed information for each sector was transmitted to each antenna of the HAP through a hybrid SDMA and NOMA. Furthermore, the HAP decodes the overlapping information of each sector. Next, an optimization problem was designed for optimal power allocation, and an optimal solution to the optimization problem was derived using the interior penalty method. According to the optimal solution, DL energy beamforming of HAP was optimally allocated by sector. Under such consideration, we maximized the sum throughput and maintained fairness in WPCN. Performance results showed that the proposed optimal power allocation for each sector using hybrid SDMA and NOMA in WPCN in terms of the sum throughput outperformed the existing equal power allocation for each sector while fairness was maintained. In summary, performance difference was about 1.4 times for sum throughput on average in the case of optimal power allocation by sector. Therefore, the hybrid approach achieved better performance.

Author Contributions: Conceptualization, J.M. and M.K.D.; methodology, J.M.; software, J.M.; validation, J.M. and M.K.D.; formal analysis, J.M.; investigation, M.K.D.; resources, J.M.; data curation, J.M. and M.K.D.; writing—original draft preparation, J.M. and M.K.D.; writing-review and editing, J.M. and M.K.D.; visualization, J.M. and M.K.D.; supervision, I.J.; project administration, I.J.; funding acquisition, I.J. All authors have read and agreed to the published version of the manuscript.

Funding: This work was partly supported by the Institute for Information and Communications Technology Promotion (IITP) grant funded by the Korean government (MSIP) (no. 2020-0-00107, development of the technology to automate the recommendations for big data analytic models that define data characteristics and problems), and partly by the National Research Foundation of Korea (NRF) grant funded by the Korean government (MSIT) (no. NRF-2019R1A2C1009894).

Data Availability Statement: The data that support the findings of this study are available on request from the corresponding author, I.J. The data are not publicly available due to their containing information that could compromise the privacy of research participants.

Conflicts of Interest: The authors declare no conflict of interest.

Sample Availability: The data for this project are confidential, but may be obtained with Data Use Agreements with the Computer Software Department of Hanyang University. Researchers interested in access to the data may contact Juhyun Maeng at jhmaeng@hanyang.ac.kr; also see http://wm.hanyang.ac.kr/xe/contact (accessed on 3 March 2022). It can take some months to negotiate data use agreements and gain access to the data. The author will assist with any reasonable replication attempts for two years following publication. 


\section{Abbreviations}

The following abbreviations are used in this manuscript:

\begin{tabular}{|c|c|}
\hline $\mathrm{ABF}$ & Analog beamforming \\
\hline ADC & Analog-to-digital converter \\
\hline AWGN & Additive white gaussian noise \\
\hline BFU & Beamforming unit \\
\hline CPE & Customer premises equipment \\
\hline CSI & Channel state information \\
\hline DAC & Digital-to-analog converter \\
\hline $\mathrm{DBF}$ & Digital beamforming \\
\hline DDC & Digital down converter \\
\hline $\mathrm{EH}$ & Energy harvesting \\
\hline HAP & Hybrid access point \\
\hline DL & Down link \\
\hline $\mathrm{HBF}$ & Hybrid beamforming \\
\hline IoT & Internet-of-Things \\
\hline LoS & Line-of-sight \\
\hline M2M & Machine-to-machine \\
\hline MIMO & Multiple-input multiple-output \\
\hline MISO & Multiple-input single-output \\
\hline MU-MISO & multiuser multi input single output \\
\hline mmWave & Mobile millimeter wave \\
\hline NOMA & Nonorthogonal Multiple Access \\
\hline OFDMA & Orthogonal frequency division multiple access \\
\hline OMA & Orthogonal multiple access \\
\hline $\mathrm{RF}$ & Radio frequency \\
\hline RFI & Radio-frequency interference \\
\hline SDMA & Space division multiple access \\
\hline SIC & Successive interference cancellation \\
\hline TDMA & Time division multiple access \\
\hline UAV & Unmanned aerial vehicle \\
\hline WD & Wireless devices \\
\hline WET & Wireless energy transfer \\
\hline WIT & Wireless information transfer \\
\hline WPCN & Wireless powered communication networks \\
\hline WPT & Wireless power transfer \\
\hline WSN & Wireless sensor networks \\
\hline
\end{tabular}

\section{References}

1. Xie, L.; Shi, Y.; Hou, Y.T.; Lou, A. Wireless power transfer and applications to sensor networks. IEEE Wirel. Commun. 2013, 20, 140-145. [CrossRef]

2. Wei, D.; Chan, H.A.; Kameri, K.V.N. Circular-Layer Algorithm for Ad Hoc Sensor Networks to Balance Power Consumption. In Proceedings of the 2006 3rd Annual IEEE Communications Society on Sensor and Ad Hoc Communications and Networks, Reston, VA, USA, 28-28 September 2006; Volume 3, pp. 945-950. [CrossRef]

3. Bi, S.; Zeng, Y.; Zhang, R. Wireless powered communication networks: An overview. IEEE Wirel. Commun. 2016, $23,10-18$. [CrossRef]

4. Bi, S.; Ho, C.K.; Zhang, R. Wireless powered communication: Opportunities and challenges. IEEE Commun. Mag. 2015, 53, 117-125. [CrossRef]

5. Desai, V.; Krzymien, L.; Sartori, P.; Xiao, W.; Soong, A.; Alkhateeb, A. Initial beamforming for mmWave communications. In Proceedings of the 2014 48th Asilomar Conference on Signals, Systems and Computers, Pacific Grove, CA, USA, 2-5 November 2014; pp. 1926-1930. [CrossRef]

6. Björnson, E.; van der perre, L.; Buzzi, S.; Larsson, E. Massive MIMO in Sub-6 GHz and mmWave: Physical, Practical, and Use-Case Differences. IEEE Wirel. Commun. 2018, 26, 100-108. [CrossRef]

7. Hampson, G.; Roberts, P.; Leach, M.; Brown, A.; Bateman, T.; Neuhold, S.; Beresford, R.; Cheng, W.; Tuthill, J.; Bunton, J.; et al. Microwave Phased Array Digital Beamforming System Design Challenges for SKA. In Proceedings of the 2015 European Microwave Conference (EuMC), Paris, France, 7-10 September 2015. [CrossRef] 
8. Hashemi, M.; Koksal, C.; Shroff, N. Energy-Efficient Power and Bandwidth Allocation in an Integrated Sub-6 GHz-Millimeter Wave System. arXiv 2017, arXiv:1710.00980.

9. Chi, K.; Chen, Z.; Zheng, K.; Zhu, Y.H.; Liu, J. Energy Provision Minimization in Wireless Powered Communication Networks With Network Throughput Demand: TDMA or NOMA? IEEE Trans. Commun. 2019, 67, 6401-6414. [CrossRef]

10. Jiang, M.; Li, Y.; Zhang, Q.; Qin, J. Joint Position and Time Allocation Optimization of UAV Enabled Time Allocation Optimization Networks. IEEE Trans. Commun. 2019, 67, 3806-3816. [CrossRef]

11. Yan, H.; Chen, Y.; Yang, S.H. Time Allocation and Optimization in UAV-enabled Wireless Powered Communication Networks IEEE Trans. Green Commun. Netw. 2021, 1. [CrossRef]

12. Koutsioumpos, M.; Zervas, E.; Hadjiefthymiades, E.; Merakos, L. Monitoring for Rare Events in a Wireless Powered Communication mmWave Sensor Network. Sensors 2020, 20, 3341. [CrossRef]

13. Shanin, N.; Garkisch, M.; Hagelauer, A.; Schober, R.; Cottatellucci, L. Optimal Resource Allocation and Beamforming for Two-User MISO WPCNs for a Non-linear Circuit-Based EH Model. arXiv 2021, arXiv:2110.01453. .

14. Lee, K.; Cho, S.; Lee, J.; Joe, I. Harvest-Then-Transceive: Throughput Maximization in Full-Duplex Wireless-Powered Communication Networks. Ieice Trans. Commun. 2018, 101, 1128-1141. [CrossRef]

15. Sun, X.; Yang, W.; Cai, Y. Secure and Reliable Transmission in mmWave NOMA Relay Networks With SWIPT. IEEE Syst. J. 2021, 1-12. [CrossRef]

16. Rouijel, A.; Hadmi, A.; Ghazi, H.E.; Mohammadi, Z. Tensor-based approach for blind separation of Interleave-NOMA 5G system. Sci. Afr. 2021, 14, e00956. [CrossRef]

17. Mobini, Z.; Mohammadi, M.; Chalise, B.K.; Suraweera, H.A.; Ding, Z. Beamforming Design and Performance Analysis of Full-Duplex Cooperative NOMA Systems. IEEE Trans. Wirel. Commun. 2019, 18, 3295-3311. [CrossRef]

18. Cao, Y.; Zhao, N.; Pan, G.; Chen, Y.; Fan, L.; Jin, M.; Alouini, M.S. Secrecy Analysis for Cooperative NOMA Networks With Multi-Antenna Full-Duplex Relay. IEEE Trans. Commun. 2019, 67, 5574-5587. [CrossRef]

19. Gau, R.H.; Chiu, H.T.; Lu, T.C. Classification-based Optimal Beamforming for NOMA Wireless Relay Networks. In Proceedings of the 2021 IEEE 93rd Vehicular Technology Conference (VTC2021-Spring), Helsinki, Finland, 25-28 April 2021; pp. 1-7. [CrossRef]

20. Wang, X.; Jia, M.; Guo, Q.; Ho, I.W.H.; Lau, F.C.M. Full-Duplex Relaying Cognitive Radio Network With Cooperative Nonorthogonal Multiple Access. IEEE Syst. J. 2019, 13, 3897-3908. [CrossRef]

21. Jiang, M.; Li, Y.; Zhang, Q.; Li, Q.; Qin, J. Secure Beamforming in Downlink MIMO Nonorthogonal Multiple Access Networks. IEEE Signal Process. Lett. 2017, 24, 1852-1856. [CrossRef]

22. Cheng, Y.; Li, K.H.; Teh, K.C.; Luo, S.; Li, B. Two-Tier NOMA-Based Wireless Powered Communication Networks. IEEE Syst. J. 2021, 1-10. [CrossRef]

23. Song, D.; Shin, W.; Lee, J.; Poor, H.V. Sum-Throughput Maximization in NOMA-Based WPCN: A Cluster-Specific Beamforming Approach. IEEE Internet Things J. 2021, 8, 10543-10556. [CrossRef]

24. Abd-Elmagid, M.A.; Biason, A.; ElBatt, T.; Seddik, K.G.; Zorzi, M. Non-Orthogonal Multiple Access schemes in Wireless Powered Communication Networks. In Proceedings of the 2017 IEEE International Conference on Communications (ICC), Paris, France, 21-25 May 2017; pp. 1-6. [CrossRef]

25. Li, F.; Wu, Y.; Nie, Y.; Shi, C. Time Allocation and Optimization in Time-Reversal Wireless Powered Communication Networks. Int. J. Antennas Propag. 2020, 2020, 8906438. [CrossRef]

26. Huang, Y.; Yang, L.; Bengtsson, M.; Ottersten, B. A limited feedback SDMA scheme with dynamic multiplexing order. In Proceedings of the 2009 IEEE 10th Workshop on Signal Processing Advances in Wireless Communications, Perugia, Italy, 21-24 June 2009; pp. 211-215. [CrossRef]

27. Gomez-Cuba, F.; Zorzi, M. Optimal link scheduling in millimeter wave multi-hop networks with space division multiple access. In Proceedings of the 2016 Information Theory and Applications Workshop (ITA), La Jolla, CA, USA, 31 January-5 February 2016; pp. 1-9. [CrossRef]

28. Yin, J.; Du, P.; Yang, G.; Zhou, H. Space-division multiple access for CDMA multiuser underwater acoustic communications. J. Syst. Eng. Electron. 2015, 26, 1184-1190. [CrossRef]

29. Chen, C.; Yang, Y.; Deng, X.; Du, P.; Yang, H. Space Division Multiple Access With Distributed User Grouping for Multi-User MIMO-VLC Systems. IEEE Open J. Commun. Soc. 2020, 1, 943-956. [CrossRef]

30. Chen, Z.; Basnayaka, D.A.; Haas, H. Space division multiple access in optical attocell networks. In Proceedings of the 2016 IEEE Wireless Communications and Networking Conference Workshops (WCNCW), Doha, Qatar, 3-6 April 2016; pp. 228-232. [CrossRef]

31. Chen, Z.; Basnayaka, D.A.; Haas, H. Space Division Multiple Access for Optical Attocell Network Using Angle Diversity Transmitters. J. Light. Technol. 2017, 35, 2118-2131. [CrossRef]

32. Bianchi, G.; Messina, D.; Scalia, L.; Tinnirello, I. A space-division time-division multiple access scheme for high throughput provisioning in WLANs. In Proceedings of the IEEE International Conference on Communications, ICC 2005, Seoul, Korea, 16-20 May 2005; Volume 4, pp. 2728-2733. [CrossRef]

33. Maeng, J.; Dahouda, M.; Joe, I. A NOMA-Combined Hybrid Approach for SDMA Improvement in Wireless Powered Communication Networks. Wirel. Pers. Commun. 2022, 122, 877-895. [CrossRef]

34. Wu, Q.; Chen, W.; Ng, D.W.K.; Schober, R. Spectral and Energy-Efficient Wireless Powered IoT Networks: NOMA or TDMA? IEEE Trans. Veh. Technol. 2018, 67, 6663-6667. [CrossRef] 
35. Rao, S.S. Geometric Programming. In Engineering Optimization; John Wiley \& Sons, Ltd.: Hoboken, NJ, USA; 2009; Chapter 8, pp. 492-543. [CrossRef]

36. Sediq, A.B.; Gohary, R.H.; Schoenen, R.; Yanikomeroglu, H. Optimal Tradeoff Between Sum-Rate Efficiency and Jain's Fairness Index in Resource Allocation. IEEE Trans. Wirel. Commun. 2013, 12, 3496-3509. [CrossRef]

37. Kunikawa, M.; Yomo, H.; Abe, K.; Ito, T. A Fair Polling Scheme for Energy Harvesting Wireless Sensor Networks. In Proceedings of the 2015 IEEE 81st Vehicular Technology Conference (VTC Spring), Glasgow, UK, 11-14 May 2015; pp. 1-5. [CrossRef]

38. Manju; Singh, S.; Kumar, S.; Nayyar, A.; Al-Turjman, F.; Mostarda, L. Proficient QoS-Based Target Coverage Problem in Wireless Sensor Networks. IEEE Access 2020, 8, 74315-74325. [CrossRef]

39. Chattopadhyay, A.K.; Bhattacharyya, C.K.; Bhattacharya, S. Single Hop Sensor Deployment Algorithm. In Proceedings of the 2012 Sixth International Conference on Sensing Technology (ICST), Kolkata, India, 18-21 December 2012; pp. 347-352. [CrossRef]

40. Amutha, J.; Sharma, S.; Nagar, J. WSN Strategies Based on Sensors, Deployment, Sensing Models, Coverage and Energy Efficiency: Review, Approaches and Open Issues. Wirel. Pers. Commun. 2020, 111, 1089-1115. [CrossRef] 